\title{
Análisis modal de oscilaciones electromecánicas en sistemas eléctricos de potencia
}

\section{Modal Analysis of Electromechanical Oscillations in Electrical Power Systems}

\author{
J.G. Calderón-Guizar \\ Gerencia de Análisis de Redes \\ Instituto de Investigaciones Eléctricas, México. \\ E-mail:jgcg@iie.org.mx
}

(Recibido: agosto de 2006; aceptado: agosto de 2007)

\begin{abstract}
Resumen
La presencia de oscilaciones electromecánicas en cualquier Sistema Eléctrico de Potencia (SEP) es una característica propia de estos sistemas. Mientras el amortiguamiento asociado con este tipo de oscilaciones se encuentre dentro de los límites considerados como aceptables para la operación continua de este tipo de sistemas, el surgimiento de éstas no se considera una amenaza para la operación segura del SEP. El presente artículo, centra su atención en la aplicación del análisis modal para evaluar el comportamiento dinámico de un SEP ante la ocurrencia de disturbios de magnitud pequeña para diferentes topologías y condiciones de operación. Los resultados reportados indican, que la aplicación del análisis modal permite la identificación directa de las causas que contribuyen en forma negativa al amortiguamiento asociado con los modos electromecánicos, así como la ubicación más adecuada de controles que contribuyan a mejorar el amortiguamiento de los mismos.
\end{abstract}

Descriptores: Análisis modal, factores de participación, oscilaciones electromecánicas, sistemasdepotencia.

\section{Abstract}

Electromechanical oscillations in any electrical power system is a typical characteristic of this kind of systems. Provided the damping associated with these oscillations lies above of a minimum specified value, the occurrence of these oscillation is not considered as a threat to the system operation. This paper focuses the attention on the application of modal analysis for assessing the dynamical behavior of a power system subjected to small disturbances for different operating conditions and transmission system topologies, as well. The reported results indicate, that modal analysis enables a straight identification of the causes that contribute negatively to the damping of the electromechanical modes.

Keywords: Modal analysis, participation factors, electromechanical oscillations, power systems. 
DOI: http://dx.doi.org/10.22201/fi.25940732e.2008.09n4.023

Análisis modal de oscilaciones electromecánicas en sistemas eléctricos de potencia

\section{Introducción}

La ocurrencia de oscilaciones electromecánicas en SEP's, es un fenómeno natural y común de este tipo de sistemas, sin importar las dimensiones de los mismos. Por esta razón, los SEP's son diseñados para que tal fenómeno no represente una amenaza para la operación continua de éstos. Sin embargo, conforme los SEP's evolucionan, tanto su topología como su política operativa también lo hacen. En las últimas cuatro décadas una gran cantidad de problemas asociados con oscilaciones en SEP's ha sido reportada en diversas partes del mundo (Kundur et al., 1996). Como resultado del análisis de estos eventos, ha sido posible identificar las siguientes coincidencias, en la mayoría de los casos, el evento a partir del cual se origina el problema no es un disturbio severo (pérdida de algún enlace importante, falla trifásica en algún punto de la red de transmisión, pérdida de un volumen importante de generación o de carga), la condición de demanda es normalmente alta y el sistema de transmisión es eléctricamente débil. En la actualidad, la industria eléctrica a nivel mundial experimenta un incremento sostenido en la demanda de energía y al mismo tiempo debe de satisfacer medidas cada vez más estrictas tanto de tipo económico como ecológico, para la construcción de nuevas líneas transmisión.

Las tendencias antes mencionadas, han provocado que los SEP's a nivel mundial operen cada vez más cerca de sus límites de seguridad durante períodos de tiempo cada vez mayores, lo que a su vez, genera las condiciones propicias para que con mayor frecuencia se presenten escenarios operativos en los cuales el fenómeno de las oscilaciones electromecánicas se torna problemático, debido principalmente al pobre amortiguamiento que exhiben dichas oscilaciones durante tales escenarios. En la operación cotidiana de todo SEP ocurre una gran variedad de eventos, como las fluctuaciones normales de carga, la salida programada de alguna unidad generadora o enlace de transmisión, los cuales pueden considerarse como disturbios de magnitud pequeña. El impacto de éstos en la operación del SEP se manifiestan en la mayoría de los casos en forma de oscilaciones con un amortiguamiento adecuado. Sin embargo, cuando el amortiguamiento asociado a dichas oscilaciones es pequeño, los disturbios antes mencionados suelen ser las causas primarias de severos problemas operativos como los reportados en Kundur et al. (1996).

El análisis del comportamiento dinámico de SEP's requiere una representación adecuada de los elementos del mismo, este requisito fundamental ocasiona que el modelo matemático resultante de un SEP sea altamente no-lineal. Sin embargo, a pesar de la naturaleza no-lineal de los SEP's el estudio de oscilaciones electromecánicas puede realizarse mediante la aplicación de técnicas para análisis de sistemas lineales, (Kundur, 1994), (Rogers 1996), (Rogers 2000) y (Yu, 1983), como lo es el análisis modal, debido a que las características de estas oscilaciones (frecuencia y amortiguamiento) están en función de la condición de operación y estructura del SEP más que de la ubicación o severidad del disturbio aplicado. En este artículo se presentan de forma breve conceptos básicos del análisis modal y su aplicación al análisis de oscilaciones electromecánicas en SEPs.

\section{Conceptos básicos de análisis modal}

El análisis modal es una metodología que permite determinar las siguientes características dinámicas: frecuencias de resonancia o modos de oscilación, amortiguamiento y el patrón asociado con la deformación del sistema o actividad relativa de las variables de estado (comúnmente denominado "mode shapes") cuando un modo en particular de una estructura es excitado. La estructura puede ser del tipo civil, mecánica, naval, aeronáutica, eléctrica, etc. (STC, 2000). El término modo, es un concepto matemático que permite describir en forma adecuada las vibraciones u oscilaciones resonantes del sistema. Los modos de un sistema pueden ser determinados tanto en forma analítica como de manera experimental. Desde un punto de vista analítico, los modos son las soluciones del conjunto de ecuaciones diferenciales que describen el comportamiento dinámico lineal de un sistema, alrededor de un estado estacionario (Richardson et al., 1997). El comportamiento dinámico de un SEP se describe en forma adecuada mediante un conjunto de ecuaciones diferenciales no-lineal, de la forma siguiente;

$$
\dot{X}=F(X, t)
$$

Con el propósito de utilizar el análisis modal para evaluar el comportamiento de los modos electromecánicos de un SEP, es necesario obtener un modelo lineal del mismo alrededor de un punto de operación determinado $\left(t_{0}\right)$. Luego entonces, el modelo linealizado del SEP puede ser expresado en forma general de la siguiente manera;

$$
\Delta \dot{X}\left(T_{o}\right)=A \Delta X\left(t_{o}\right)
$$


DOI: http://dx.doi.org/10.22201/fi.25940732e.2008.09n4.023

J.G. Calderón-Guizar

Donde $\boldsymbol{A}$ es la matriz de estado del sistema y $\Delta \boldsymbol{X}$ es el vector de las variables de estado del sistema.

Una vez que se ha obtenido un modelo matemático del SEP como el representado por la ecuación (2), es posible evaluar su comportamiento dinámico mediante el análisis de los eigenvalores y eigenvectores de la matriz de estado del sistema.

\section{Eigenvalores}

Los eigenvalores de la matriz de estado $\boldsymbol{A}$ son las raíces $\left(\lambda_{\mathbf{i}}\right)$ del polinomio característico asociado con la matriz de estado, el cual está definido en forma compacta como (Kundur, 1994);

$$
\operatorname{det}\left(A-\lambda_{i} I\right)=0
$$

Los valores de $\lambda_{\mathbf{i}}$ que satisfacen a la ecuación (3) pueden ser reales o complejos y cuando la matriz $\boldsymbol{A}$ es real como en el caso de la matriz de estado de un SEP, las raíces complejas siempre se presentan en pares conjugados.

\section{Eigenvectores ("Mode Shapes")}

Los eigenvectores son vectores asociados con cada uno de los eigenvalores $\lambda_{\mathbf{i}}$ que satisfacen el siguiente sistema de ecuaciones;

$$
\begin{aligned}
& A u_{i}=\lambda_{i} u_{i} \\
& v_{i} A=\lambda_{i} v_{i}
\end{aligned}
$$

A cada uno de estos vectores $\boldsymbol{u}_{\boldsymbol{i}}$ y $\boldsymbol{v}_{\boldsymbol{i}}$ se les conoce como eigenvectores derechos o "mode shapes" e izquierdos, respectivamente. Los elementos de los eigenvectores derechos dan una medida de la actividad relativa de las variables de estado cuando un modo en particular es excitado.

En otras palabras, el k-ésimo elemento del eigenvector $\boldsymbol{u}_{\boldsymbol{i}}$ mide la actividad de la variable de estado $\boldsymbol{x}_{\boldsymbol{k}}$ en el i-ésimo eigenvalor, mientras que el $\mathbf{k}$-ésimo elemento del eigenvector $\boldsymbol{v}_{\boldsymbol{i}}$ es una medida de la influencia de dicha actividad sobre el i-ésimo eigenvalor.

\section{Factores de participación}

Puesto que la magnitud de los elementos de los eigenvectores son influenciadas por las unidades de las variables de estado, el uso de esos valores para medir la influencia de las variables de estado sobre un eigenvalor es más bien limitado.

Los factores de participación definidos como el producto de las $\mathbf{j}$-ésimas componentes de los eigenvectores derecho $\left(\boldsymbol{u}_{j i}\right)$ e izquierdo $\left(\boldsymbol{v}_{\boldsymbol{i j}}\right)$ asociados con el i-ésimo eigenvalor, esto es:

$$
P_{j i}=u_{j i} v_{i j}
$$

son magnitudes adimensionales, es decir, son independientes de las unidades asociadas con las variables de estado. Estos factores son una medida de la participación relativa de la j-ésima variable de estado en el i-ésimo eigenvalor y viceversa, lo cual permite de manera confiable determinar cuáles son las variables de estado que mayor influencia neta tienen sobre cualquiera de los eigenvalores del sistema.

\section{Descripción del sistema de estudio y resultados de las simulaciones}

Para propósitos del presente artículo, se emplea la representación más simple de un SEP, conocida como Sistema Máquina Bus-Infinito (SMBI). Esta representación del SEP consiste en un generador síncrono conectado a través de un transformador y una línea de doble circuito a un bus-infinito, como se muestra en las figuras 1 y 2 .

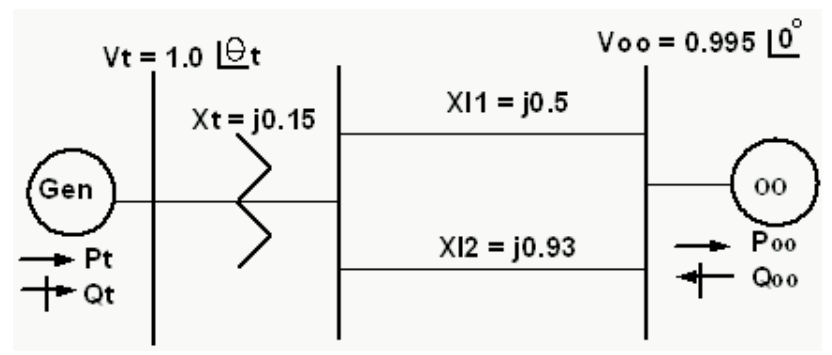

Figura 1. Representación gráfica del sistema de estudio y parámetros del sistema de transmisión en pu

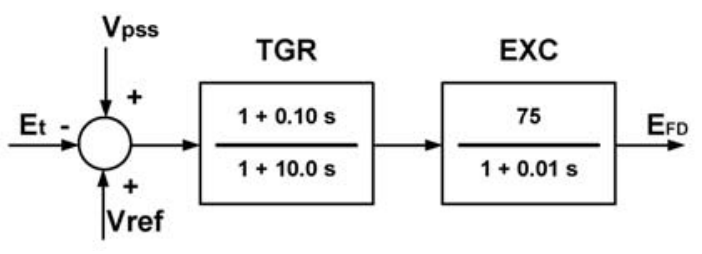

Figura 2. Diagrama de bloques y parámetros del sistema de excitación

Con el propósito de evaluar la influencia de la topología del sistema de transmisión, así como la condición de 
operación (las diferentes condiciones de operación consideradas se presentan en la tabla 1). Sobre el comportamiento de los modos electromecánicos del SEP bajo estudio se realizaron las siguientes simulaciones:

Simulación 1. El generador entrega 1110 MW a través del transformador y el doble circuito.

Simulación 2. El generador entrega $1110 \mathrm{MW}$ a través del transformador y del circuito Xl1, en este caso el circuito Xl2 se consideró fuera de servicio.

Simulación 3. El generador entrega $1554 \mathrm{MW}$ a través del transformador y el doble circuito.

Simulación 4. El generador entrega $1554 \mathrm{MW}$ a través del transformador y del circuito X11, en este caso el circuito X12 se consideró fuera de servicio.

La magnitud de los voltajes en terminales del generador y del bus infinito en las cuatro condiciones de operación analizadas se mantuvieron en 1.0 pu y 0.995 pu respectivamente, como se muestra en la figura 1.

Los resultados de las simulaciones antes descritas se presentan en forma condensada en la tabla 2, en ella se muestran la frecuencia de oscilación en $\mathrm{Hz}$ y el amortiguamiento en \%, asociado con los modos electromecánicos del SEP analizado para cada una de las condiciones de operación consideradas en este artículo. El análisis de los resultados presentados en la tabla 2 , permite inferir que conforme se incrementa la potencia eléctrica de salida del generador, el amortiguamiento asociado con los modos electromecánicos se reduce de manera considerable, aún y cuando la topología del sistema de transmisión no se modifique entre una condición de operación y otra. Estos resultados también indican que si la potencia eléctrica de salida del generador no varía pero la topología del sistema de transmisión se modifica de manera tal que el sistema de transmisión resultante sea menos robusto eléctricamente (impedancias de transmisión mayores) el amortiguamiento asociado con los modos electromecánicos del sistema se reduce también en forma importante. En las simulaciones realizadas se presentó el caso en que al aumentar la potencia eléctrica de salida del generador en un $20 \%$ y perder uno de los enlaces de transmisión (simulación 4) respecto al caso base (simulación 1) el amortiguamiento de dichos modos se torna negativo, lo cual indica que para tal escenario, la condición de operación del SEP será inestable. Todos los resultados reportados en el presente artículo fueron obtenidos usando el paquete PacDyn (CEPEL, 2005).

Tabla 1. Descripción de las diferentes condiciones de operación consideradas

\begin{tabular}{|c|c|c|c|c|c|}
\hline $\begin{array}{c}\text { Condición de Operación } \\
\text { asociada con }\end{array}$ & $\begin{array}{c}\theta t \\
\text { grados }\end{array}$ & $\begin{array}{l}\mathrm{Pt} \\
\mathrm{MW}\end{array}$ & $\begin{array}{l}\text { Ot } \\
\text { MVAR }\end{array}$ & $\begin{array}{l}\mathrm{P} \infty \\
\mathrm{MW}\end{array}$ & $\begin{array}{l}Q_{\infty} \\
\text { MVAR }\end{array}$ \\
\hline Simulación 1 & 13.8146 & 1110.0 & 157.828 & 1110.0 & 111.225 \\
\hline Simulación 2 & 19.0645 & 1110.0 & 203.470 & 1110.0 & 169.401 \\
\hline Simulación 3 & 19.5296 & 1554.0 & 290.798 & 1554.0 & 244.196 \\
\hline Simulación 4 & 27.2121 & 1554.0 & 393.184 & 1554.0 & 359.135 \\
\hline
\end{tabular}

Tabla 2. Frecuencia de oscilación y amortiguamiento asociados con los modos electromecánicos del SEP, para las diferentes condiciones de operación analizadas

\begin{tabular}{ccc}
\hline Simulación & Frecuencia de Oscilación $(\mathrm{Hz})$ & Amortiguamiento $(\%)$ \\
\hline 1 & 1.2488 & 5.0375 \\
2 & 1.1264 & 3.0357 \\
3 & 1.2527 & 1.3270 \\
4 & 1.1072 & -1.2542 \\
\hline
\end{tabular}


DOI: http://dx.doi.org/10.22201/fi.25940732e.2008.09n4.023

J.G. Calderón-Guizar

\section{Conclusiones}

A partir de los resultados obtenidos de las diferentes simulaciones realizadas se puede concluir lo siguiente con relación al amortiguamiento de los modos electromecánicos de un SEP:

- Un incremento en la potencia eléctrica de salida del generador reduce el amortiguamiento asociado a los modos electromecánicos del sistema. Esto es, entre mayor es la demanda que satisface un SEP, menor es el amortiguamiento asociado con los modos electromecánicos.

- Un incremento en la impedancia del sistema de transmisión, sistemas de transmisión débiles, contribuye a una reducción importante del amortiguamiento de los modos electromecánicos del SEP.

- El límite de estabilidad de un SEP se reduce considerablemente conforme el sistema de transmisión se torna eléctricamente débil. En el caso considerado en este artículo, el sistema es incapaz de soportar una carga de 1554.0 MW cuando el circuito Xl2 se encuentra fuera de servicio. Mientras este circuito se encuentre en operación el sistema es capaz de soportar dicha carga.

- Tanto el incremento en la carga del sistema como la disminución en la robustez del sistema de transmisión de un SEP afectan de manera negativa el amortiguamiento asociado con los modos electromecánicos del SEP.

\section{Referencias}

CEPEL. PacDyn User's Manual. CEPEL Centro de Pesquisas de Energia Elétrica. 2005.

Kundur P. Power System Stability and Control. The EPRI Power System Engineering Series. McGraw Hill. 1994. Pp. 699-825.

Kundur P. et al. Utility Practice and Experience with Power System Oscillations. In: Analysis and Control of Power System Oscillations (CIGRE Task Force 38.01.07), 1996. Pp. 2:1-3:8 CIGRE.

Rogers G. Demistifying Power System Oscillations. IEEE Computer Applications in Power. 1996.

Rogers G. Power System Oscillations. Kluwer Academic Publishers. 2000. Pp. 7-73.

Richardson M. et al. Is It Mode Shape, or an Operating Deflection Shape? Sound \& Vibration Magazine, 30 th Anniversary Issue, 1997, pp. 1-11.

STC. What is modal analysis? [on linea], 2000. Available on: http://www.structuraltechnology.com/modal_analysis. htm

Yu N.Y. Electric Power System Dynamics. Academic Press. New Cork. 1983. Pp. 66-96.

\section{Semblanza del autor}

Jorge Guillermo Calderón-Guizar. Obtuvo el título de ingeniero industrial electricista en 1983 por el Instituto Tecnológico de Morelia, y en 1986, el grado de maestro en ciencias en ingeniería eléctrica en la Sección de Graduados de la ESIME-IPN, y en 1995, el doctorado en filosofía (PhD) en ingeniería eléctrica en el Instituto de Ciencia y Tecnología de la Universidad de Manchester (UMIST) en el Reino Unido. Es investigador de la Gerencia de Análisis de Redes del Instituto de Investigaciones Eléctricas (IIE) desde 1985. Sus áreas de interés son el análisis dinámico y estático de sistemas eléctricos de potencia (estabilidad de pequeñas señales, transitoria, estabilidad de voltaje y flujos de carga en sistemas de potencia de gran escala e industriales). Ha participado en proyectos de consultoría del IIE al AMM de Guatemala. Ha sido profesor de tiempo parcial en licenciatura y postgrado en diferentes Universidades del Estado de Morelos. Es revisor de la publicación IEEE Transactions on Power Systems. Fue presidente del Capítulo de Potencia de la Sección Morelos del IEEE durante el período 2002-2003 y presidente de la Sección Morelos del IEEE durante el período 2005-2006. En el año 2001 el IEEE le otorgó el grado de Senior Member. 